\title{
Article \\ Stability of Interval Type-3 Fuzzy Controllers for Autonomous Vehicles
}

\author{
Man-Wen Tian ${ }^{1}$, Shu-Rong Yan ${ }^{1}$, Ardashir Mohammadzadeh ${ }^{2}{ }^{\oplus}$, Jafar Tavoosi ${ }^{3}{ }^{(0}$, Saleh Mobayen ${ }^{4, *} \mathbb{C}$, \\ Rabia Safdar ${ }^{5}\left(\mathbb{D}\right.$, Wudhichai Assawinchaichote ${ }^{6, *} \mathbb{B}$, Mai The Vu ${ }^{7, *} \mathbb{\infty}$ and Anton Zhilenkov ${ }^{8}$
}

\section{check for}

updates

Citation: Tian, M.-W.; Yan, S.-R.; Mohammadzadeh, A.; Tavoosi, J.; Mobayen, S.; Safdar, R.; Assawinchaichote, W.; Vu, M.T.; Zhilenkov, A. Stability of Interval Type-3 Fuzzy Controllers for Autonomous Vehicles. Mathematics 2021, 9, 2742. https://doi.org/ $10.3390 /$ math 9212742

Academic Editor: Vassilis C. Gerogiannis

Received: 4 October 2021

Accepted: 24 October 2021

Published: 28 October 2021

Publisher's Note: MDPI stays neutral with regard to jurisdictional claims in published maps and institutional affiliations.

Copyright: (c) 2021 by the authors. Licensee MDPI, Basel, Switzerland. This article is an open access article distributed under the terms and conditions of the Creative Commons Attribution (CC BY) license (https:/ / creativecommons.org/licenses/by/ $4.0 /)$.
1 National Key Project Laboratory, Jiangxi University of Engineering, Xinyu 338000, China; manwen@126.com (M.-W.T.); yjy848@126.com (S.-R.Y.)

2 Electrical Engineering Department, University of Bonab, Bonab 5551395133, Iran; a.mzadeh@ubonab.ac.ir

3 Department of Electrical Engineering, Ilam University, Ilam 69315516, Iran; j.tavoosi@ilam.ac.ir

4 Future Technology Research Center, National Yunlin University of Science and Technology, Douliu 64002, Taiwan

5 Department of Mathematics, Lahore College Women University, Lahore 54000, Pakistan; rabia.safdar1109@gmail.com

6 Department of Electronic and Telecommunication Engineering, Faculty of Engineering, King Mongkut's University of Technology Thonburi, Bangkok 10140, Thailand

7 School of Intelligent Mechatronics Engineering, Sejong University, Seoul 05006, Korea

8 Department of Cyber-Physical Systems, St. Petersburg State Marine Technical University, 190121 Saint-Petersburg, Russia; zhilenkovanton@gmail.com

* Correspondence: mobayens@yuntech.edu.tw (S.M.); wudhichai.asa@kmutt.ac.th (W.A.); maithevu90@sejong.ac.kr (M.T.V.)

Abstract: Economic efficient Autonomous Road Vehicles (ARVs) are invariably subjected to uncertainties and perturbations. Therefore, control of vehicle systems requires stability to withstand the effect of variations in the nominal performance. Lateral path-tracking is a substantial task of ARVs, especially in critical maneuvering and cornering with variable speed. In this study, a new controller on the basis of interval type-3 (T3) fuzzy logic system (FLSs) is designed. The main novelties and advantages are as follows. (1) The uncertainty is a main challenge in the path-following problem of ARVs. However, in the fuzzy-based approaches, the bounds of uncertainty are assumed to be known. However, in the our suggested approach, the bounds of uncertainties are also fuzzy sets and type- 3 FLSs with online adaptation rules are suggested to handle the uncertainties. (2) The approximation errors (AEs) and perturbations are investigated and tackled by the compensators. (3) The bounds of estimation errors are also uncertain and are estimated by the suggested adaptation laws. (4) The stability is ensured under unknown dynamics, perturbations and critical maneuvers. (5) Comparison with the benchmarking techniques and conventional fuzzy approaches verifies that the suggested path-following scheme results in better maneuver performance.

Keywords: fuzzy system; autonomous vehicles; machine learning; path-following; stability

\section{Introduction}

New technologies are surrounding the lives of many people in the world day by day. Meanwhile, the global automotive industry is also trying to keep pace with the latest technology in the world and surpass its competitors. Driverless vehicles are a kind of automatic vehicle that can move people or vehicles like conventional cars today. Driverless cars have a new technology that a prominent feature of this type of technology is that a significant part of the car control is out of human hands and computers actually control the car. These types of vehicles include a complex set of sensors, radars and scanners that can communicate all environmental and internal conditions in the most vital detail to their powerful processors, so that those processors can control the vehicle [1-4]. 
The path-following problem of ARVs is a challengeable control problem, because of various dynamic perturbations such as variation of longitudinal speed, smoothness and roughness of road, road adhesion, critical maneuver and so on [5]. This problem has got a remarkable attention and some controllers have been designed. For example, in [6], the undershoot response of conventional path-following control approaches is analyzed and the effect of initial values of control parameters is investigated. The estimation of unmeasurable states of ARV is studied in [7] and an observer-based scheme is designed for path-following. The dynamic surface controller is developed in [8] by the use of event-triggered scheme and the accuracy of the path tracking is compared with other controllers. In [9], the velocities are estimated, an adaptive control strategy is schemed and the boundedness of signals are proved. In [10], by the use of Lotka-Volterra equations, a dynamic model is extracted and an optimal controller is designed for parking management problem. In [11], the problem of lane-change motion is studied and the reduction of control effort and improvement of ride quality are ensured by a predictive controller. The actuator faults are investigated in [12], and by the use of composite energy function, the stability is analyzed. In [13], by Barrier function an optimal controller is suggested, and the problem of energy consumption and passenger safety are investigated. The global course constraint is considered in [14], and by the use of gain shaping approach a feedback controller is designed. In [15], a fault-tolerant controller is developed by the use of $\mathrm{H}_{\infty}$ criteria, and the stability is analyzed under actuator faults. In [16], the problem of obstacle avoidance is taken into account, and a path-following scheme is designed by the predictive control approach. In [17], the problem of energy consumption is investigated, and an energyefficient controller is designed for path-following. The yaw-moment control strategy is suggested in [18], and by the simulations the accuracy improvement is evaluated.

One of the main challenging in path-following problem is the existence of noisy information and high level of uncertainties $[19,20]$. To improve the robustness of the conventional controllers against uncertainties, the fuzzy-based controllers have also been developed [21-24]. For example, in [25], a type-2 (T2) FLS-based path-following system is suggested, and it is shown that the maximum of tracking error of desired path is $\sim 0.04 \mathrm{~m}$. In [26], the velocity is modeled by a FLS, and it is managed by the fuzzy model. In [27], the effect of uncertainties are analyzed, and it is shown that FLSs improve the lane-change maneuver performance. In [28], the FLSs are used to estimate the states, and it is combined with the sliding mode approach to construct a path-following scheme. In [29], the FLSs are used to adjust the gains of PID controller, and the handling stability is investigated. In [30], the reinforcement learning approach is presented to optimize the a FLS-based controller, and the problem of overtaking is studied. The Takagi-Sugeno FLSs are used in [31] to cope with uncertainties, and by designing the sliding mode controller, the yaw stability is analyzed. The FLS-based predictive controller is presented in [32] to determine the best front wheel angle. In [33], a FLS-based path-following approach is constructed, and the stability is analyzed in the presence of various disturbances. The steering angle controller is designed in [34] using optimized FLSs, and the convergence problem is analyzed.

Recently, the interval type-3 FLSs have been developed to increase the estimation ability of type-2 FLSs. In various studies, it has been shown that the IT3FLSs have better performance in the presence of high noisy and uncertain environments such as power control problem [35], energy management [36], solving equations [37], gyroscope systems [38], forecasting problem [39] and so on. However, type-3 FLS-based controllers have not been developed for ARVs with high uncertain dynamics.

Regarding above discussion, we propose a new controller based on IT3FLSs. The dynamics of ARV are online modeled by IT3FLSs. The IT3FLSs are tuned by adaptive learning algorithm. The adaptive supervisors are also compensate the estimation errors and perturbations. The stability and robustness are analyzed and ensured by the designed adaptation laws and compensators. The well path tracking performance of designed controller is verified for double-lane-change (DLC) maneuver under variable disturbances, unknown 
dynamics and various speed. The comparison with other basic controllers and type- 2 FLS-based controllers show that the designed scheme well improves the maneuver quality.

\section{Problem Formulation}

As depicted in Figure 1, the bicycle model of ARV is considered. The objective is to design a controller for the heading angle (yaw angle) such that the yaw stability to be guaranteed and the rotating of vehicle to be prevented. Furthermore, the yaw velocity should be approached to the desired value. As given in Figure 1, the lateral error represents the nearest interspace between ARV and favorable path, $\phi$ denotes the yaw rate, $\phi_{d}$ is the desired level of $\phi, \phi_{e}$ is the error between $\phi$ and $\phi_{d}$ and $\eta$ is the ARV heading angle. The error dynamics can be written as

$$
\left\{\begin{array}{c}
\phi_{e}=\phi-\phi_{d}=\phi-\vartheta_{\chi} / R(\rho) \\
\dot{y}=\vartheta_{\chi} \sin (\eta)+\vartheta_{y} \cos (\eta) \\
\dot{\eta}=\phi
\end{array}\right.
$$

where $R(\rho)$ is the curvature radius as depicted in Figure 1, and $\rho$ is the arc length. To follow the desired trajectory, the variables $\phi_{e}$ and $\dot{y}$ should be converged to zero by the designed controller. The dynamics of yaw plane ARV can be written as

$$
\left\{\begin{array}{c}
\dot{\vartheta}_{y}=\frac{1}{m}\left(F_{y f}+F_{y r}\right)-\vartheta_{\chi} \phi \\
\dot{\phi}=\frac{1}{I_{z}}\left(F_{y f} l_{f}-F_{y r} l_{r}+\Delta T\right)
\end{array}\right.
$$

where $\vartheta_{\chi}-\vartheta_{y}$ are the longitudinal-lateral velocities, $y-\dot{y}$ are the displacement/velocity, $\eta-\dot{\eta}$ are the heading angle-yaw rates, $\Delta T$ denotes yaw moment, $F_{y f}-F_{y r}$ are the lateral tire force, $l_{f}-l_{r}$ denote the distance between the central gravity and front-rear wheels, $m$ and $I_{z}$ are the mass and mass-moment of inertia. The external yaw moment is written as

$$
\Delta T=\sum_{i} \sum_{j=1}^{2}(-1)^{j} F_{x i j} \frac{l_{b}}{2} \quad i=f, r
$$

The lateral forces $\omega_{f}$ and $\omega_{r}$ are written as follows:

$$
F_{y f}=\omega_{f} \xi_{f}, \quad F_{y r}=\omega_{r} \xi_{r}
$$

The cornering characteristics can be considered as

$$
\omega_{f}=\tilde{\omega}_{f}+\Delta \omega_{f}, \quad \omega_{r}=\tilde{\omega}_{r}+\Delta \omega_{r}
$$

where $\tilde{\omega}_{f} / \tilde{\omega}_{r}$ are the cornering stiffness for front/rear tires. $\Delta \omega_{f}$ and $\Delta \omega_{r}$ denote the uncertainties. The side slip angles of front/rear tires are described as

$$
\left\{\begin{array}{c}
\xi_{f}=\tan ^{-1}\left[\frac{\vartheta_{\chi} \sin (\zeta)+l_{f} \phi}{\vartheta_{\chi} \cos (\zeta)}\right]-\delta_{f} \\
\xi_{r}=\tan ^{-1}\left[\frac{\vartheta_{\chi} \sin (\zeta)-l_{r} \phi}{\vartheta_{\chi} \cos (\zeta)}\right]
\end{array}\right.
$$

where $\delta_{f} / \zeta$ denote the steering/sideslip angle $\left(\zeta \approx \vartheta_{y} / \vartheta_{\chi}\right)$. From (3)-(6) and (1)-(2), we can derive 


$$
\left\{\begin{aligned}
\dot{y}= & \vartheta_{\chi} \sin (\eta)+\vartheta_{y} \cos (\eta) \\
\dot{\vartheta}_{y}= & \frac{\omega_{f}}{m}\left\{\tan ^{-1}\left[\frac{\vartheta_{\chi} \sin (\zeta)+l_{f} \phi}{\vartheta_{\chi} \cos (\zeta)}\right]-\delta_{f}\right\} \\
& +\frac{\omega_{r}}{m}\left\{\tan ^{-1}\left[\frac{\vartheta_{\chi} \sin (\zeta)-l_{r} \phi}{\vartheta_{\chi} \cos (\zeta)}\right]\right\}-\vartheta_{\chi} \phi \\
\dot{\phi}= & \frac{1}{I_{z}}\left(\left\{l_{f} \omega_{f} \tan ^{-1}\left[\frac{\vartheta_{\chi} \sin (\zeta)+l_{f} \phi}{\vartheta_{\chi} \cos (\zeta)}\right]-\delta_{f}\right\}\right. \\
& \left.+\left\{l_{r} \omega_{r} \tan ^{-1}\left[\frac{\vartheta_{\chi} \sin (\zeta)-l_{r} \phi}{\vartheta_{\chi} \cos (\zeta)}\right]\right\}+\Delta T\right)
\end{aligned}\right.
$$

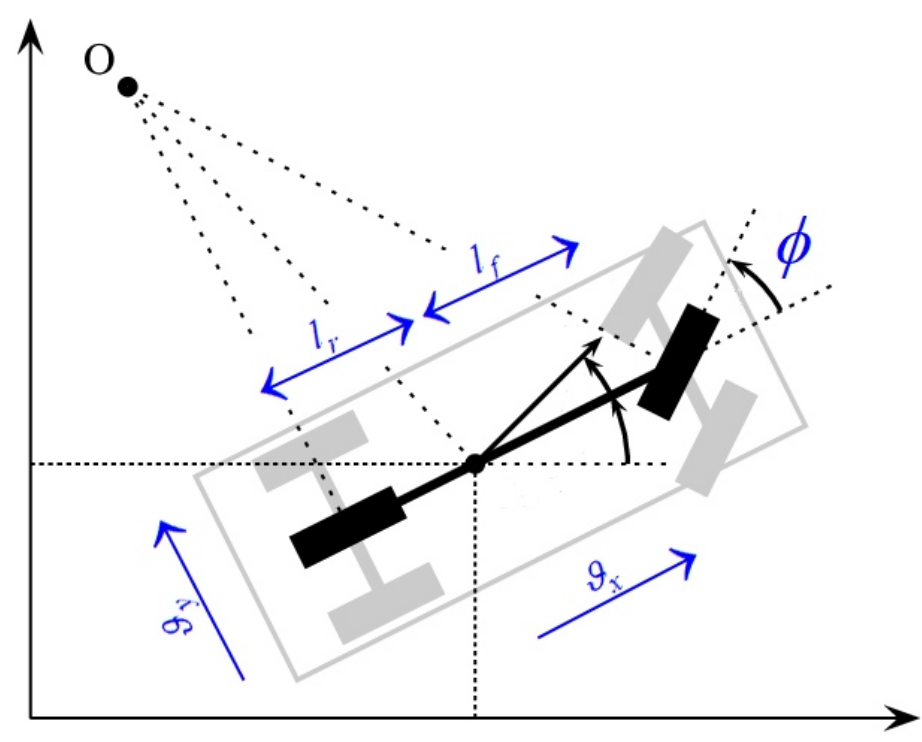

Figure 1. The yaw-plane model.

The dynamics are rewritten as follows:

$$
\begin{aligned}
& \dot{\chi}_{1}=g_{1}(\underline{\chi})+\kappa_{1}(\underline{\chi}) u_{1} \\
& \dot{\chi}_{2}=g_{2}(\underline{\chi})+\kappa_{2}(\underline{\chi}) u_{2}
\end{aligned}
$$

where $\chi_{1}=\vartheta_{y}, \chi_{2}=\phi, u_{1}=\delta_{f}, u_{2}=\Delta T, \kappa_{1}=\frac{1}{I_{z}}, \kappa_{2}=\frac{\omega_{f}}{m}$ and

$$
\begin{gathered}
g_{1}(\underline{\chi})=\frac{\omega_{f}}{m}\left\{\tan ^{-1}\left[\frac{\vartheta_{\chi} \sin (\zeta)+l_{f} \phi}{\vartheta_{\chi} \cos (\zeta)}\right]\right\} \\
+\frac{\omega_{r}}{m}\left\{\tan ^{-1}\left[\frac{\vartheta_{\chi} \sin (\zeta)-l_{r} \phi}{\vartheta_{\chi} \cos (\zeta)}\right]\right\}-\vartheta_{\chi} \phi \\
g_{2}(\underline{\chi})=\frac{1}{I_{z}}\left\{l_{f} \omega_{f} \tan ^{-1}\left[\frac{\vartheta_{\chi} \sin (\zeta)+l_{f} \phi}{\vartheta_{\chi} \cos (\zeta)}\right]-\delta_{f}\right\} \\
+\left\{l_{r} \omega_{r} \tan ^{-1}\left[\frac{\vartheta_{\chi} \sin (\zeta)-l_{r} \phi}{\vartheta_{\chi} \cos (\zeta)}\right]\right\}
\end{gathered}
$$

The general diagram of the suggested control approach is shown in Figure 2. 


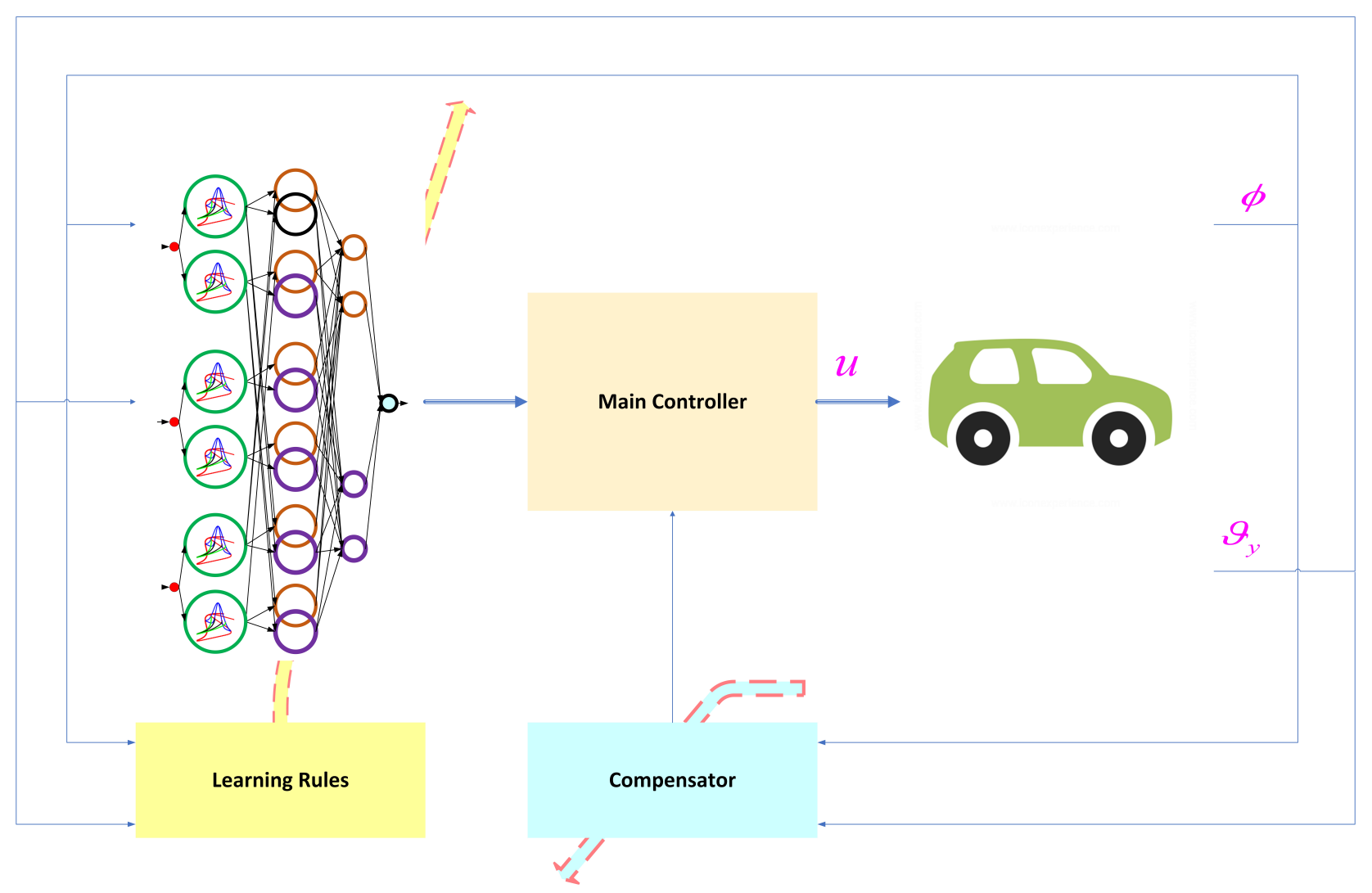

Figure 2. The general diagram of the suggested control scheme.

\section{Type-3 FLS}

The IT3FLSs have been developed to improve the estimation ability of conventional type-2 FLSs [40]. In this paper, new tuning rules are suggested for online learning. As shown in Figure 2, IT3FLSs are used to estimate the nonlinear functions, $g_{1}, g_{2}, \kappa_{1}$ and $\kappa_{2}$. The structure of $\hat{g}_{1}$ and $\hat{\kappa}_{1}$ are shown in Figure 3. The structure of $\hat{g}_{2}$ and $\hat{\kappa}_{2}$ are similar to $\hat{g}_{1}$ and $\hat{\kappa}_{1}$ with difference that in $\hat{g}_{2}$ and $\hat{\kappa}_{2}$ the inputs variables are $\phi, \vartheta$ and $u_{1}$. The computations are illustrated in below:

(1) The inputs of $\hat{g}_{1}$ and $\hat{\kappa}_{1}$ are $\phi$ and $\vartheta$ and the inputs of $\hat{g}_{2}$ and $\hat{\kappa}_{2}$ are $\phi, \vartheta$ and $u_{1}$.

(2) The suggested membership functions (MFs) are shown in Figure 4. Two MFs are considered for inputs $\phi, \vartheta$ and $u_{1}$. The centers of Gaussian MFs correspond to the upper bound (UB) and lower bound (LB) of inputs. The upper MF is denoted by $\bar{\Lambda}$ and the lower MF is denoted by $\underline{\Lambda}$. For $\phi$, the upper memberships of $\bar{\Lambda}_{\phi}$ and $\underline{\Lambda}_{\phi}$ for the upper slice $\bar{A}_{i}$ and lower slices $\underline{A}_{i}$ are

$$
\begin{aligned}
& \bar{\mu}_{\underline{\Lambda}_{\phi \mid \bar{A}_{i}}}(\phi)=\exp \left(-\frac{\left(\phi-C_{\underline{\Lambda}_{\phi}}\right)^{2}}{\bar{\sigma}_{\underline{\Lambda}_{\phi \mid \bar{A}_{i}}}^{2}}\right) \\
& \bar{\mu}_{\underline{\Lambda}_{\phi \mid \underline{A}_{i}}}(\phi)=\exp \left(-\frac{\left(\phi-C_{\underline{\Lambda}_{\phi}}\right)^{2}}{\bar{\sigma}_{\underline{\Lambda}_{\phi \mid \underline{A}_{i}}}^{2}}\right) \\
& \bar{\mu}_{\bar{\Lambda}_{\phi \mid \bar{A}_{i}}}(\phi)=\exp \left(-\frac{\left(\phi-C_{\bar{\Lambda}_{\phi}}\right)^{2}}{\bar{\sigma}_{\bar{\Lambda}_{\phi \mid \bar{A}_{i}}}^{2}}\right)
\end{aligned}
$$


Mathematics 2021, 9, 2742

6 of 17

$$
\bar{\mu}_{\bar{\Lambda}_{\phi \mid \underline{A}_{i}}}(\phi)=\exp \left(-\frac{\left(\phi-C_{\bar{\Lambda}_{\phi}}\right)^{2}}{\bar{\sigma}_{\bar{\Lambda}_{\phi \mid \underline{A}_{i}}}^{2}}\right)
$$

Similarly, the lower memberships are obtained as

$$
\begin{gathered}
\underline{\mu}_{\underline{\Lambda}_{\phi \mid \bar{A}_{i}}}(\phi)=\exp \left(-\frac{\left(\phi-C_{\underline{\Lambda}_{\phi}}\right)^{2}}{\underline{\sigma}_{\underline{\Lambda}_{\phi \mid \bar{A}_{i}}}^{2}}\right) \\
\underline{\mu}_{\underline{\Lambda}_{\phi \mid \underline{A}_{i}}}(\phi)=\exp \left(-\frac{\left(\phi-C_{\underline{\Lambda}_{\phi}}\right)^{2}}{\underline{\sigma}_{\underline{\Lambda}_{\phi \mid \underline{A}_{i}}}^{2}}\right) \\
\underline{\mu}_{\bar{\Lambda}_{\phi \mid \bar{A}_{i}}}(\phi)=\exp \left(-\frac{\left(\phi-C_{\bar{\Lambda}_{\phi}}\right)^{2}}{\underline{\sigma}_{\bar{\Lambda}_{\phi \mid \bar{A}_{i}}}}\right) \\
\underline{\mu}_{\bar{\Lambda}_{\phi \mid \underline{A}_{i}}}(\phi)=\exp \left(-\frac{\left(\phi-C_{\bar{\Lambda}_{\phi}}\right)^{2}}{\underline{\sigma}_{\bar{\Lambda}_{\phi \mid \underline{A}_{i}}}^{2}}\right)
\end{gathered}
$$

where $C_{\underline{\Lambda}_{\phi}}$ and $C_{\bar{\Lambda}_{\phi}}$ are the centers of $\underline{\Lambda}_{\phi}$ and $\bar{\Lambda}_{\phi}$, respectively. $\bar{\sigma}_{\underline{\Lambda}_{\phi \mid \underline{A}_{i}}}$ and $\bar{\sigma}_{\underline{\Lambda}_{\phi \mid \bar{A}_{i}}}^{2}$ are the upper width of $\underline{\Lambda}_{\phi}$ at upper slice $\bar{A}_{i}$ and lower slices $\underline{A}_{i}$. For input $\vartheta$, we have

$$
\begin{aligned}
& \bar{\mu}_{\underline{\Lambda}_{\vartheta \mid \bar{A}_{i}}}(\vartheta)=\exp \left(-\frac{\left(\vartheta-C_{\underline{\Lambda}_{\vartheta}}\right)^{2}}{\bar{\sigma}_{\underline{\Lambda}_{\vartheta \mid \bar{A}_{i}}}^{2}}\right) \\
& \bar{\mu}_{\underline{\Lambda}_{\vartheta \mid \underline{A}_{i}}}(\vartheta)=\exp \left(-\frac{\left(\vartheta-C_{\underline{\Lambda}_{\vartheta}}\right)^{2}}{\bar{\sigma}_{\underline{\Lambda}_{\vartheta \mid \underline{A}_{i}}}^{2}}\right) \\
& \bar{\mu}_{\bar{\Lambda}_{\vartheta \mid \bar{A}_{i}}}(\vartheta)=\exp \left(-\frac{\left(\vartheta-C_{\bar{\Lambda}_{\vartheta}}\right)^{2}}{\bar{\sigma}_{\bar{\Lambda}_{\vartheta \mid \bar{A}_{i}}}^{2}}\right) \\
& \bar{\mu}_{\bar{\Lambda}_{\vartheta \mid \underline{A}_{i}}}(\vartheta)=\exp \left(-\frac{\left(\vartheta-C_{\bar{\Lambda}_{\vartheta}}\right)^{2}}{\bar{\sigma}_{\bar{\Lambda}_{\vartheta \mid \underline{A}_{i}}}^{2}}\right)
\end{aligned}
$$

Similarly, the lower memberships are obtained as

$$
\begin{aligned}
& \underline{\mu}_{\underline{\Lambda}_{\vartheta \mid \bar{A}_{i}}}(\vartheta)=\exp \left(-\frac{\left(\vartheta-C_{\underline{\Lambda}_{\vartheta}}\right)^{2}}{\underline{\sigma}_{\underline{\Lambda}_{\vartheta \mid \bar{A}_{i}}}^{2}}\right) \\
& \underline{\mu}_{\underline{\Lambda}_{\vartheta \mid \underline{A}_{i}}}(\vartheta)=\exp \left(-\frac{\left(\vartheta-C_{\underline{\Lambda}_{\vartheta}}\right)^{2}}{\underline{\sigma}_{\underline{\Lambda}_{\vartheta \mid \underline{A}_{i}}}^{2}}\right) \\
& \underline{\mu}_{\bar{\Lambda}_{\vartheta \mid \bar{A}_{i}}}(\vartheta)=\exp \left(-\frac{\left(\vartheta-C_{\bar{\Lambda}_{\vartheta}}\right)^{2}}{\underline{\sigma}_{\bar{\Lambda}_{\vartheta \mid \bar{A}_{i}}^{2}}^{2}}\right)
\end{aligned}
$$


Mathematics 2021, 9, 2742

7 of 17

$$
\underline{\mu}_{\bar{\Lambda}_{\vartheta \mid \underline{A}_{i}}}(\vartheta)=\exp \left(-\frac{\left(\vartheta-C_{\bar{\Lambda}_{\vartheta}}\right)^{2}}{\underline{\sigma}_{\bar{\Lambda}_{\vartheta \mid A_{i}}}^{2}}\right)
$$

where $C_{\underline{\Lambda}_{\vartheta}}$ and $C_{\bar{\Lambda}_{\vartheta}}$ are the centers of $\underline{\Lambda}_{\vartheta}$ and $\bar{\Lambda}_{\vartheta}$, respectively. $\bar{\sigma}_{\underline{\Lambda}_{\vartheta \mid A_{i}}}$ and $\bar{\sigma}_{\underline{\Lambda}_{\vartheta \mid \bar{A}_{i}}}^{2}$ are the upper width of $\underline{\Lambda}_{\vartheta}$ at upper slice $\bar{A}_{i}$ and lower slices $\underline{A}_{i}$.

(3) The output of $\hat{g}$ is written as

$$
\hat{g}_{i}=\underline{\alpha}_{i}^{T} \varphi_{i}
$$

where $\underline{\alpha}_{i}$ and $\varphi_{i}$ are

$$
\begin{aligned}
\alpha_{i} & =\left[\underline{\omega}_{i 1}, \ldots, \underline{\omega}_{i R}, \bar{\omega}_{i 1}, \ldots, \bar{\omega}_{i R}\right]^{T} \\
\varphi_{i} & =\left[\underline{\varphi}_{i 1}, \ldots, \underline{\varphi}_{i R^{\prime}}, \bar{\varphi}_{i 1}, \ldots, \bar{\varphi}_{i R}\right]^{T}
\end{aligned}
$$

where $R$ is the rule numbers, $\overline{\boldsymbol{\omega}}_{i l}$ and $\underline{\omega}_{i l}$ represent the $l-$ th rule parameters. $\underline{\varphi}_{i l}$ and $\bar{\varphi}_{i l}$ are

$$
\begin{aligned}
& \bar{\varphi}_{l}=\frac{\sum_{j=1}^{n_{A}} \bar{A}_{j} \frac{\bar{\theta}_{\mu_{s}=\bar{A}_{j}}}{\sum_{l=1}^{R}\left(\bar{\theta}^{l} \mu_{s}=\bar{A}_{j}+\underline{\theta}_{\mu_{s}}=\bar{A}_{j}\right)}}{\sum_{j=1}^{n_{A}}\left(\bar{A}_{j}+\underline{A}_{j}\right)} \\
& +\frac{\sum_{j=1}^{n_{A}} \underline{A}_{j} \frac{\bar{\theta}_{\mu_{s}=\underline{A}_{j}}^{l}}{\sum_{l=1}^{R}\left(\bar{\theta}_{\mu_{s}=\underline{A}_{j}}^{l}+\underline{\theta}_{\mu_{s}}^{l}=\underline{A}_{j}\right)}}{\sum_{j=1}^{n_{A}}\left(\bar{A}_{j}+\underline{A}_{j}\right)}, l=1, \ldots, R \\
& \underline{\varphi}_{l}=\frac{\sum_{j=1}^{n_{A}} \bar{A}_{j} \frac{\underline{\theta}_{\mu_{s}=\bar{A}_{j}}}{\sum_{l=1}^{R}\left(\bar{\theta}_{\mu_{s}=\bar{A}_{j}}+\underline{\theta}_{\mu_{s}=\bar{A}_{j}}^{l}\right)}}{\sum_{j=1}^{n_{A}}\left(\bar{A}_{j}+\underline{A}_{j}\right)} \\
& +\frac{\sum_{j=1}^{n_{A}} \underline{A}_{j} \frac{\underline{\theta}_{\mu_{s}=\underline{A}_{j}}^{l}}{\sum_{l=1}^{R}\left(\bar{\theta}_{\mu_{s}}^{l}=\underline{A}_{j}+\underline{\theta}_{\mu_{s}}^{l}=\underline{A}_{j}\right)}}{\sum_{j=1}^{n_{A}}\left(\bar{A}_{j}+\underline{A}_{j}\right)}, l=1, \ldots, R
\end{aligned}
$$

where $n_{A}$ denotes slices numbers and

$$
\begin{aligned}
& \underline{\varphi}_{l}=\frac{\sum_{j=1}^{n_{A}} \bar{A}_{j} \frac{\underline{\theta}_{\mu_{s}=\bar{A}_{j}}^{l}}{\sum_{l=1}^{R}\left(\bar{\theta}_{\mu_{s}=\bar{A}_{j}}+\underline{\theta}_{\mu_{s}=\bar{A}_{j}}^{l}\right)}}{\sum_{j=1}^{n_{A}}\left(\bar{A}_{j}+\underline{A}_{j}\right)}
\end{aligned}
$$

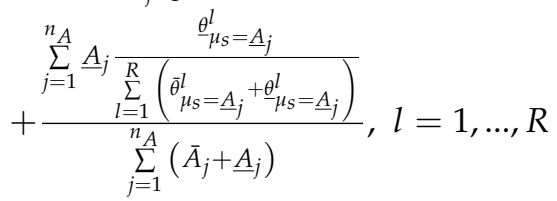

$$
\begin{aligned}
& \underline{\theta}_{\mu_{s}=\bar{A}_{j}}^{l}=\underline{\mu}_{\Lambda_{\phi} \mid \bar{A}_{j}}(\phi) \underline{\mu}_{\Lambda_{\vartheta} \mid \bar{A}_{j}}(\vartheta) \underline{\mu}_{\Lambda_{u_{1}} \mid \bar{A}_{j}}\left(u_{1}\right) \\
& \underline{\theta}_{\mu_{s}=\underline{A}_{j}}^{l}=\underline{\mu}_{\Lambda_{\phi} \mid \underline{A}_{j}}(\phi) \underline{\mu}_{\Lambda_{\vartheta} \mid \underline{A}_{j}}(\vartheta) \underline{\mu}_{\Lambda_{u_{1}} \mid \underline{A}_{j}}\left(u_{1}\right) \\
& \bar{\theta}_{\mu_{s}=\bar{A}_{j}}^{l}=\bar{\mu}_{\Lambda_{\phi} \mid \bar{A}_{j}}(\phi) \bar{\mu}_{\Lambda_{\vartheta} \mid \bar{A}_{j}}(\vartheta) \bar{\mu}_{\Lambda_{u_{1} \mid \bar{A}_{j}}}\left(u_{1}\right) \\
& \bar{\theta}_{\mu_{s}=\underline{A}_{j}}^{l}=\bar{\mu}_{\Lambda_{\phi} \mid \underline{A}_{j}}(\phi) \bar{\mu}_{\Lambda_{\vartheta} \mid \underline{A}_{j}}(\vartheta) \bar{\mu}_{\Lambda_{u_{1}} \mid \underline{A}_{j}}\left(u_{1}\right)
\end{aligned}
$$


where the $l-t h$ rule is given as

$$
\begin{aligned}
& \text { Rule\#l : If } \phi \text { is } \Lambda_{\varphi \mid A_{j}} \text { and } \vartheta \text { is } \Lambda_{\vartheta \mid A_{j}} \\
& \text { and } u_{1} \text { is } \Lambda_{u_{1} \mid A_{j}} \text { Then } \hat{g} \in\left[\underline{\mathscr{Q}}_{l}, \overline{\boldsymbol{\omega}}_{l}\right]
\end{aligned}
$$

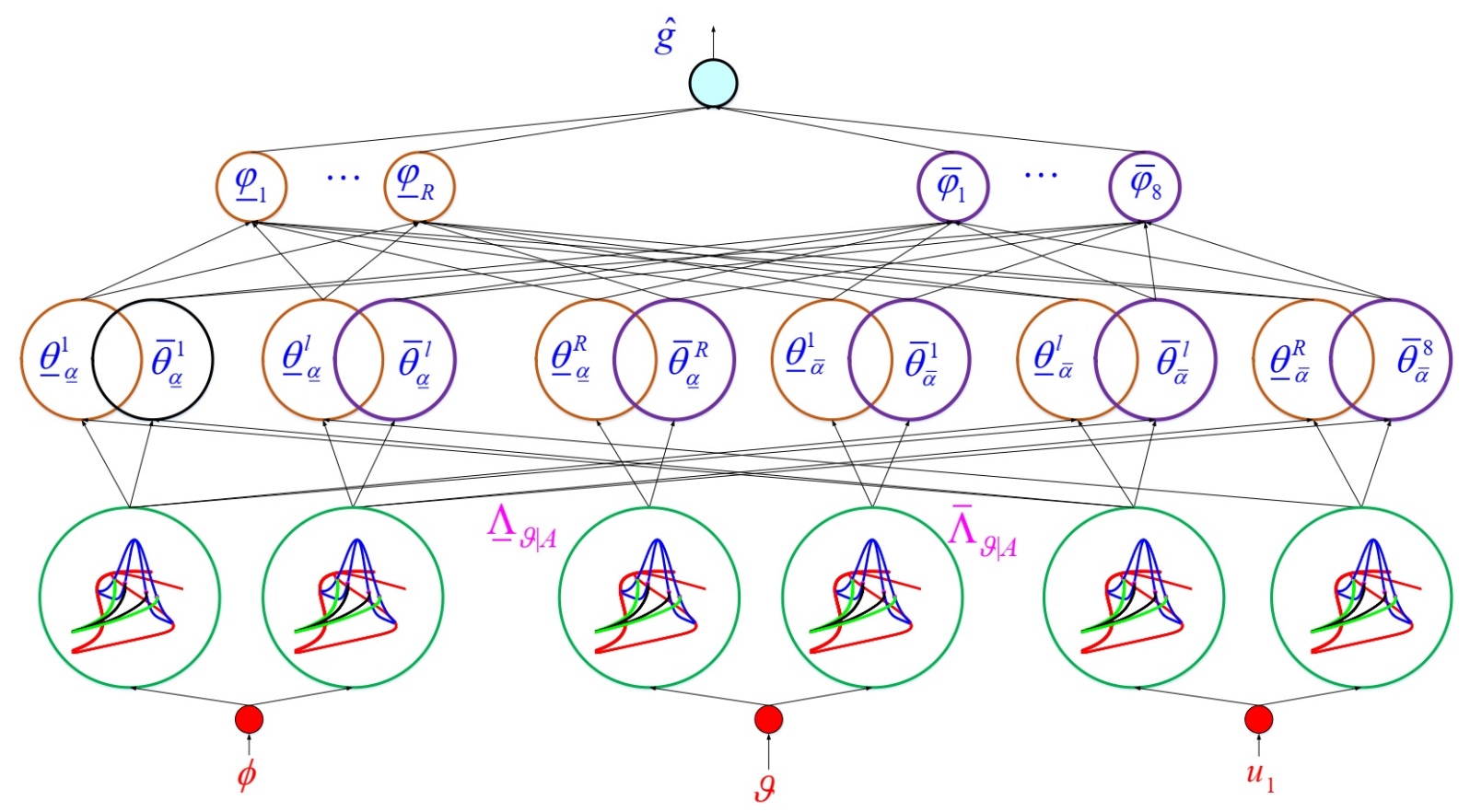

Figure 3. Structure of IT3-FLS.

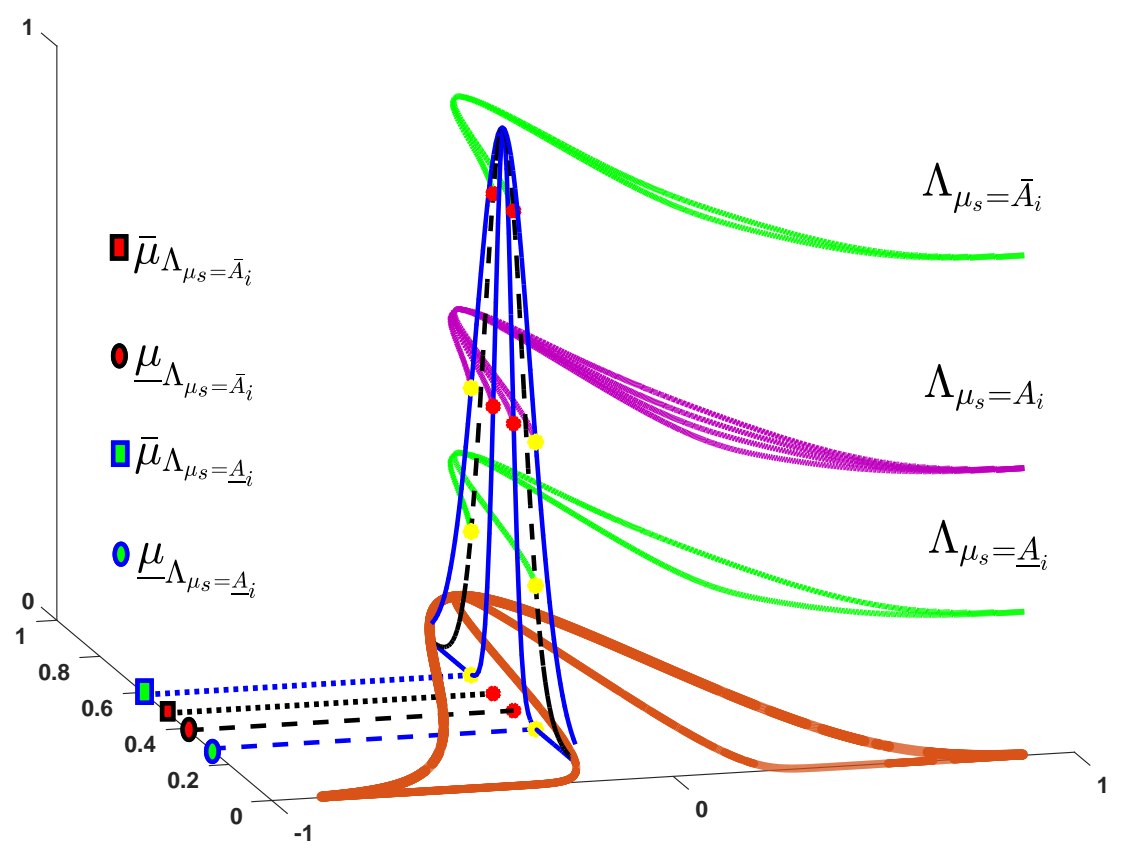

Figure 4. The type-3 MF.

\section{Main Reults}

The dynamics of ARV (8) are approximated as

$$
\begin{aligned}
& \dot{\hat{\chi}}_{1}=\hat{g}_{1}\left(\underline{w}_{1} \mid \underline{\alpha}_{1}\right)+\hat{\kappa}_{1}\left(\underline{w}_{1} \mid \underline{\beta}_{1}\right) u_{1} \\
& \dot{\hat{\chi}}_{2}=\hat{g}_{2}\left(\underline{w}_{2} \mid \underline{\alpha}_{2}\right)+\hat{\kappa}_{2}\left(\underline{w}_{1} \mid \underline{\beta}_{2}\right) u_{2}
\end{aligned}
$$


where $\hat{\chi}_{1} / \hat{\chi}_{2}$ are the estimations of $\chi_{1} / \chi_{2}, \hat{g}_{1} / \hat{g}_{2}$ and $\hat{\kappa}_{1} / \hat{\kappa}_{2}$ are the suggested IT3FLSs. $\underline{\alpha}_{1} / \underline{\alpha}_{2}$ and $\underline{\beta}_{1} / \underline{\beta}_{2}$ are the tunable parameters of $\hat{g}_{1} / \hat{g}_{2}$ and $\kappa_{1} / \kappa_{2}$, respectively. $\underline{w}_{1}$ and $\underline{w}_{2}$ are

$$
\begin{aligned}
& \underline{w}_{1}=\left[\chi_{1}, \chi_{2}\right]^{T} \\
& \underline{w}_{2}=\left[\chi_{1}, \chi_{2}, u_{1}\right]^{T}
\end{aligned}
$$

From (8) and (34), the errors $\tilde{\chi}_{1}=\chi_{1}-\hat{\chi}_{1}$ and $\tilde{\chi}_{2}=\chi_{2}-\hat{\chi}_{2}$ are written as

$$
\begin{aligned}
\dot{\tilde{\chi}}_{1} & =g_{1}(\underline{\chi})-\hat{g}_{1}\left(\underline{w}_{1} \mid \underline{\alpha}_{1}\right) \\
& +\left(\kappa_{1}-\hat{\kappa}_{1}\left(\underline{w}_{1} \mid \underline{\beta}_{1}\right)\right) u_{1} \\
\dot{\tilde{\chi}}_{2} & =g_{2}(\underline{\chi})-\hat{g}_{2}\left(\underline{w}_{2} \mid \underline{\alpha}_{2}\right) \\
& +\left(\kappa_{2}-\hat{\kappa}_{2}\left(\underline{w}_{1} \mid \underline{\beta}_{2}\right)\right) u_{2}
\end{aligned}
$$

Considering the optimal values as $\underline{\alpha}_{i}^{*}$ and $\underline{\beta}_{i}^{*}$ from (36), we have

$$
\begin{aligned}
\dot{\tilde{\chi}}_{1} & =\hat{g}_{1}\left(\underline{w}_{1} \mid \underline{\alpha}_{1}^{*}\right)-\hat{g}_{1}\left(\underline{w}_{1} \mid \underline{\alpha}_{1}\right) \\
& +\left(\hat{\kappa}_{1}\left(\underline{w}_{1} \mid \underline{\beta}_{1}^{*}\right)-\hat{\kappa}_{1}\left(\underline{w}_{1} \mid \underline{\beta}_{1}\right)\right) u_{1} \\
& +g_{1}(\underline{\chi})-\hat{g}_{1}\left(\underline{w}_{1} \mid \underline{\alpha}_{1}^{*}\right) \\
& +\left(\kappa_{1}-\hat{\kappa}_{1}\left(\underline{w}_{1} \mid \underline{\beta}_{1}^{*}\right)\right) u_{1} \\
\dot{\tilde{\chi}}_{2} & =\hat{g}_{2}\left(\underline{w}_{2} \mid \underline{\alpha}_{2}^{*}\right)-\hat{g}_{2}\left(\underline{w}_{2} \mid \underline{\alpha}_{2}\right) \\
& +\left(\hat{\kappa}_{2}\left(\underline{w}_{2} \mid \underline{\beta}_{2}^{*}\right)-\hat{\kappa}_{1}\left(\underline{w}_{2} \mid \underline{\beta}_{2}\right)\right) u_{2} \\
& +g_{2}(\underline{\chi})-\hat{g}_{2}\left(\underline{w}_{2} \mid \underline{\alpha}_{2}^{*}\right) \\
& +\left(\kappa_{2}-\hat{\kappa}_{2}\left(\underline{w}_{2} \mid \underline{\beta}_{2}^{*}\right)\right) u_{2}
\end{aligned}
$$

The approximation errors $Q_{1}$ and $Q_{2}$ are defined as follows:

$$
\begin{array}{r}
Q_{1}=g_{1}(\underline{\chi})-\hat{g}_{1}\left(\underline{w}_{1} \mid \underline{\alpha}_{1}^{*}\right) \\
+\left(\kappa_{1}-\hat{\kappa}_{1}\left(\underline{w}_{1} \mid \underline{\beta}_{1}^{*}\right)\right) u_{1} \\
Q_{2}=g_{2}(\underline{\chi})-\hat{g}_{2}\left(\underline{w}_{2} \mid \underline{\alpha}_{2}^{*}\right) \\
\quad+\left(\kappa_{2}-\hat{\kappa}_{2}\left(\underline{w}_{2} \mid \underline{\beta}_{2}^{*}\right)\right) u_{2}
\end{array}
$$

From (39) and (40), we obtain

$$
\begin{aligned}
\dot{\tilde{\chi}}_{1} & =\hat{g}_{1}\left(\underline{w}_{1} \mid \underline{\alpha}_{1}^{*}\right)-\hat{g}_{1}\left(\underline{w}_{1} \mid \underline{\alpha}_{1}\right) \\
& +\left(\hat{\kappa}_{1}\left(\underline{w}_{1} \mid \underline{\beta}_{1}^{*}\right)-\hat{\kappa}_{1}\left(\underline{w}_{1} \mid \underline{\beta}_{1}\right)\right) u_{1} \\
& +Q_{1} \\
\dot{\tilde{\chi}}_{2} & =\hat{g}_{2}\left(\underline{w}_{2} \mid \underline{\alpha}_{2}^{*}\right)-\hat{g}_{2}\left(\underline{w}_{2} \mid \underline{\alpha}_{2}\right) \\
& +\left(\hat{\kappa}_{2}\left(\underline{w}_{2} \mid \underline{\beta}_{2}^{*}\right)-\hat{\kappa}_{2}\left(\underline{w}_{2} \mid \underline{\beta}_{2}\right)\right) u_{2} \\
& +Q_{2}
\end{aligned}
$$

Considering vector form (27), we have

$$
\begin{aligned}
& \dot{\tilde{\chi}}_{1}=\underline{\tilde{\alpha}}_{1}^{T} \varphi_{1}+Q_{1}+\underline{\tilde{\beta}}_{1}^{T} \varphi_{1} u_{1} \\
& \dot{\tilde{\chi}}_{2}=\underline{\tilde{\alpha}}_{2}^{T} \varphi_{2}+Q_{2}+\underline{\tilde{\beta}}_{2}^{T} \varphi_{2} u_{2}
\end{aligned}
$$

where

$$
\underline{\tilde{\alpha}}_{i}=\underline{\tilde{\alpha}}_{i}^{*}-\underline{\tilde{\alpha}}_{i}, i=1,2
$$




$$
\underline{\tilde{\beta}}_{i}=\underline{\tilde{\beta}}_{i}^{*}-\underline{\tilde{\beta}}_{i}, i=1,2
$$

The errors $Q_{i}, i=1,2$ are written as $Q_{i}=q_{i} e_{i} . q_{i}, i=1,2$ are estimated as $\hat{q}_{i}, i=1,2$.

Theorem 1. By control signals (47), the compensator (48) and adaptation rules (49) and (50), the asymptotic stability of system (8) is ensured.

$$
\begin{gathered}
u_{1}=\frac{1}{\kappa_{1}}\left(-\hat{g}_{1}\left(\underline{w}_{1} \mid \underline{\alpha}_{1}\right)+\dot{r}_{1}-\iota_{1} e_{1}+u_{c 1}\right) \\
u_{2}=\frac{1}{\kappa_{2}}\left(-\hat{g}_{2}\left(\underline{w}_{2} \mid \underline{\alpha}_{2}\right)+\dot{\gamma}_{2}-\iota_{2} e_{2}+u_{c 2}\right) \\
u_{c 1}=-\tilde{\chi}_{1} \hat{q}_{1} \\
u_{c 2}=-\tilde{\chi}_{2} \hat{q}_{2} \\
\underline{\alpha}_{1}=\gamma \tilde{\chi}_{1} \varphi_{1} \\
\underline{\dot{\alpha}}_{2}=\gamma \tilde{\chi}_{2} \varphi_{2} \\
\dot{\dot{\beta}}_{1}=\gamma \tilde{\chi}_{1} \varphi_{1} u_{1} \\
\dot{\dot{\beta}}_{2}=\gamma \tilde{\chi}_{2} \varphi_{2} u_{2} \\
\dot{\hat{q}}_{1}=\gamma \tilde{\chi}_{1} e_{1} \\
\dot{\hat{q}}_{2}=\gamma \tilde{\chi}_{2} e_{2}
\end{gathered}
$$

where $r_{i}, i=1,2$ are reference signals. $\iota_{1}$ and $\iota_{2}$ denote constants. $u_{c 1}$ and $u_{c 2}$ denote the compensators. $e_{1}=\hat{\chi}_{1}-r_{1}$ and $e_{2}=\hat{\chi}_{2}-r_{2}$ represent tracking errors, $\tilde{\chi}_{1}$ and $\tilde{\chi}_{2}$ are defined as $\tilde{\chi}_{1}=\chi_{1}-\hat{\chi}_{1}, \tilde{\chi}_{2}=\chi_{2}-\hat{\chi}_{2}$ and $\gamma$ is a constant between 0 and 1 .

Proof. From (34) and (47), we can write

$$
\begin{aligned}
& \dot{e}_{1}=-\iota_{1} e_{1}+u_{c 1} \\
& \dot{e}_{2}=-\iota_{2} e_{2}+u_{c 2}
\end{aligned}
$$

Consider the Lyapunov function as

$$
\begin{aligned}
& \vartheta=\frac{1}{2} e_{1}^{2}+\frac{1}{2} e_{2}^{2} \\
& \quad+\frac{1}{2} \tilde{\chi}_{1}^{2}+\frac{1}{2} \tilde{\chi}_{2}^{2} \\
& \quad+\frac{1}{2 \gamma} \underline{\tilde{\alpha}}_{1}^{T} \tilde{\underline{\alpha}}_{1}+\frac{1}{2 \gamma} \tilde{\underline{\alpha}}_{2}^{T} \tilde{\underline{\alpha}}_{2} \\
& \quad+\frac{1}{2 \gamma} \tilde{\beta}_{1}^{T} \tilde{\beta}_{1}+\frac{1}{2 \gamma} \tilde{\beta}_{2}^{T} \tilde{\beta}_{2} \\
& +\frac{1}{2 \gamma} \tilde{q}_{1}^{2}+\frac{1}{2 \gamma} \tilde{q}_{2}^{2}
\end{aligned}
$$

where

$$
\tilde{q}_{i}=q_{i}-\hat{q}_{i}
$$

By taking the time derivative of $\vartheta$ in (52), we obtain

$$
\begin{aligned}
& \dot{\vartheta}=e_{1} \dot{e}_{1}+e_{2} \dot{e}_{2} \\
& \quad+\tilde{\chi}_{1} \dot{\tilde{\chi}}_{1}+\tilde{\chi}_{2} \dot{\tilde{\chi}}_{2} \\
& \quad-\frac{1}{\gamma} \underline{\underline{\alpha}}_{1}^{T} \underline{\dot{\alpha}}_{1}-\frac{1}{\gamma} \tilde{\alpha}_{2}^{T} \underline{\alpha}_{2} \\
& \quad-\frac{1}{\gamma} \tilde{\beta}_{1}^{T} \dot{\beta}_{1}-\frac{1}{\gamma} \tilde{\beta}_{2}^{T} \dot{\beta}_{2} \\
& -\frac{1}{\gamma} \tilde{q}_{1} \underline{\hat{q}}_{1}-\frac{1}{\gamma} \tilde{q}_{2} \underline{\hat{q}}_{2}
\end{aligned}
$$

Considering $\dot{e}_{i}$ and $\tilde{\chi}_{i}, i=1,2$, from (44) and (51), $\dot{\vartheta}$ becomes 


$$
\begin{aligned}
\dot{\vartheta} & =e_{1}\left(-\iota_{1} e_{1}+u_{c 1}\right)+e_{2}\left(-\iota_{2} e_{2}+u_{c 2}\right) \\
& +\tilde{\chi}_{1}\left(\underline{\tilde{\alpha}}_{1}^{T} \varphi_{1}+Q_{1}+\underline{\tilde{\beta}}_{1}^{T} \varphi_{1} u_{1}\right) \\
& +\tilde{\chi}_{2}\left(\underline{\tilde{\alpha}}_{2}^{T} \varphi_{2}+Q_{2}+\underline{\tilde{\beta}}_{2}^{T} \varphi_{2} u_{2}\right) \\
& -\frac{1}{\gamma} \underline{\tilde{\alpha}}_{1}^{T} \dot{\alpha}_{1}-\frac{1}{\gamma} \underline{\tilde{\alpha}}_{2}^{T} \underline{\dot{\alpha}}_{2} \\
& -\frac{1}{\gamma} \tilde{\tilde{\beta}}_{1}^{T} \dot{\dot{\beta}}_{1}-\frac{1}{\gamma} \tilde{\tilde{\beta}}_{2}^{T} \dot{\dot{\beta}}_{2} \\
& -\frac{1}{\gamma} \tilde{q}_{1} \dot{\hat{q}}_{1}-\frac{1}{\gamma} \tilde{q}_{2} \dot{\hat{q}}_{2}
\end{aligned}
$$

Simplifications of (55), yields

$$
\begin{aligned}
\dot{\vartheta} & =-\iota_{1} e_{1}^{2}+e_{1} u_{c 1}-\iota_{2} e_{2}^{2}+e_{2} u_{c 2} \\
& +\underline{\tilde{\alpha}}_{1}^{T}\left(\tilde{\chi}_{1} \varphi_{1}-\frac{1}{\gamma} \underline{\dot{\alpha}}_{1}\right)+\tilde{\chi}_{1} Q_{1} \\
& +\underline{\tilde{\alpha}}_{2}^{T}\left(\tilde{\chi}_{2} \varphi_{2}-\frac{1}{\gamma} \underline{\dot{\alpha}}_{2}\right)+\tilde{\chi}_{2} Q_{2} \\
& +\underline{\tilde{\beta}}_{1}^{T}\left(\tilde{\chi}_{1} \varphi_{1} u_{1}-\frac{1}{\gamma} \underline{\dot{\beta}}_{1}\right) \\
& +\tilde{\tilde{\beta}}_{2}^{T}\left(\tilde{\chi}_{2} \varphi_{2} u_{2}-\frac{1}{\gamma} \dot{\dot{\beta}}_{2}\right) \\
& -\frac{1}{\gamma} \tilde{q}_{1} \dot{\hat{q}}_{1}-\frac{1}{\gamma} \tilde{q}_{2} \dot{\hat{q}}_{2}
\end{aligned}
$$

Considering the tuning rules as (49) and (50), we obtain

$$
\begin{aligned}
\dot{\vartheta}= & -\iota_{1} e_{1}^{2}-\iota_{2} e_{2}^{2} \\
& +\tilde{\chi}_{1} Q_{1}+e_{1} u_{c 1} \\
& +\tilde{\chi}_{2} Q_{2}+e_{2} u_{c 2} \\
& -\frac{1}{\gamma} \tilde{q}_{1} \dot{\hat{q}}_{1}-\frac{1}{\gamma} \tilde{q}_{2} \dot{\hat{q}}_{2}
\end{aligned}
$$

Considering $Q_{i}$ as $Q_{i}=q_{i} e_{i}, i=1,2$ and by some simplifications, we have

$$
\begin{aligned}
& \dot{\vartheta}=-\iota_{1} e_{1}^{2}-\iota_{2} e_{2}^{2} \\
& +\tilde{\chi}_{1} \tilde{q}_{1} e_{1}+e_{1} u_{c 1}+\tilde{\chi}_{1} \hat{q}_{1} e_{1} \\
& +\tilde{\chi}_{2} \tilde{q}_{2} e_{2}+e_{2} u_{c 2}+\tilde{\chi}_{2} \hat{q}_{2} e_{2} \\
& -\frac{1}{\gamma} \tilde{q}_{1} \dot{\hat{q}}_{1}-\frac{1}{\gamma} \tilde{q}_{2} \dot{\hat{q}}_{2}
\end{aligned}
$$

Equation (58) can be rewritten as

$$
\begin{aligned}
& \dot{\vartheta}=-\iota_{1} e_{1}^{2}-\iota_{2} e_{2}^{2} \\
& +\tilde{q}_{1}\left(\tilde{\chi}_{1} e_{1}-\frac{1}{\gamma} \dot{\hat{q}}_{1}\right)+e_{1} u_{c 1}+\tilde{\chi}_{1} \hat{q}_{1} e_{1} \\
& +\tilde{q}_{2}\left(\tilde{\chi}_{2} e_{2}-\frac{1}{\gamma} \dot{\hat{q}}_{2}\right)+e_{2} u_{c 2}+\tilde{\chi}_{2} \hat{q}_{2} e_{2}
\end{aligned}
$$

Then, from the tuning laws as $\dot{\hat{q}}_{1}=\gamma \tilde{\chi}_{1} e_{1}$ and $\dot{\hat{q}}_{2}=\gamma \tilde{\chi}_{2} e_{2}$, we can write

$$
\begin{aligned}
\dot{\vartheta}= & -\iota_{1} e_{1}^{2}-\iota_{2} e_{2}^{2} \\
& +e_{1} u_{c 1}+\tilde{\chi}_{1} \hat{q}_{1} e_{1} \\
& +e_{2} u_{c 2}+\tilde{\chi}_{2} \hat{q}_{2} e_{2}
\end{aligned}
$$

Then if $u_{c 1}$ and $u_{c 2}$ are considered as $u_{c 1}=-\tilde{\chi}_{1} \hat{q}_{1}$ and $u_{c 2}=-\tilde{\chi}_{2} \hat{q}_{2}, \dot{v}$, is written as:

$$
\dot{\vartheta}=-\iota_{1} e_{1}^{2}-\iota_{2} e_{2}^{2}
$$

By the use of Barbalat's lemma, it can be shown that $\lim _{t \rightarrow \infty} e_{1}(t) \rightarrow 0$ and $\lim _{t \rightarrow \infty} e_{2}(t) \rightarrow 0$. From Barbalat's lemma, it should be proved that $e_{1} \in \ell^{2}$ and $e_{2} \in \ell^{2}$. From (61), we have 


$$
\begin{gathered}
-\int_{0}^{t} \dot{\vartheta}(v) d v=\vartheta(0)-\vartheta(t) \\
\leq \vartheta(0)<\infty
\end{gathered}
$$

From (62), it can be written as

$$
\iota_{1} \int_{0}^{t} e_{1}^{2}(v) d v+\iota_{2} \int_{0}^{t} e_{2}^{2}(v) d v<\infty
$$

and

$$
\begin{aligned}
& \int_{0}^{t} e_{1}^{2}(v) d v<\infty \\
& \int_{0}^{t} e_{2}^{2}(v) d v<\infty
\end{aligned}
$$

Then, $e_{1} \in \ell^{2}$ and $e_{2} \in \ell^{2}$, and the asymptotically stability is proved.

\section{Simulations}

The capability of the suggested controller is examined under various conditions. The parameters of the case study system are given in Table 1 . The reference are considered as [41]

$$
\begin{aligned}
& r_{1}=\frac{d}{d t} \tan ^{-1}\left\{4.05\left(\frac{1}{\cosh (\bar{p})}\right)^{2} \frac{1.2}{25}\right. \\
& \left.-5.7\left(\frac{1}{\cosh (\bar{q})}\right)^{2}\left(\frac{1.2}{21.95}\right)\right\} \\
& \begin{array}{c}
r_{2}=\frac{d}{d t}\left\{\frac{4.02}{2}(1+\tanh (\bar{p}))-\right. \\
\left.\frac{5.7}{2}(1+\tanh (\bar{q}))\right\}
\end{array}
\end{aligned}
$$

where $\bar{p}=2.40(v t-27.29) / 26-1.20, \bar{q}=2.5(v t-56.47) / 22.95-1.20$ and $\vartheta_{x}=10 \mathrm{~m} / \mathrm{s}$.

The performance of changing of heading angle and lateral displacement are depicted in Figure 5. We see that the ARV track the desired path with good accuracy. The ARV heading angle is changed between $t=3-9 s$, and we see a desired lateral displacement. The tracking error performance is shown in Figure 6. We observe that in the lane changing, the biggest error is less than $0.07 \mathrm{~m}$. The heading error is also less than $\pm 0.006 \mathrm{rad}$ that show the stability. The control signals are shown in Figure 7. We observe that, the designed controller results in the good control signals such that the biggest magnitude is less 2 rad and $5000 \mathrm{~N} \cdot \mathrm{m}$ and they are stabilized in a finite time. It should be noted that in the onset of motion the suggested IT3FLSs have not been tuned. After fast learning of IT3FLSs, the trajectories are quickly converged to the desired range.

The accuracy is numerically investigated in contrast to the methods such as active disturbance-rejection controller (ADRC) [42], T2 fuzzy controller (T2FLC) [33], FLS-based predictive controller (FLS-MPC) [43] and linear-quadratic tracking (LQT). The values of the RMS and error peaks are given in Table 2. We see that the results for the suggested controller is better than T2FLC and other benchmarking controllers.

Table 1. Simulation parameters.

\begin{tabular}{ccc}
\hline Parameter & Value & Unit \\
\hline$\omega_{f}$ & 67,600 & $\mathrm{~N} / \mathrm{rad}$ \\
$I_{z}$ & 2352 & $\mathrm{~kg} \cdot \mathrm{m}^{2}$ \\
$l_{r}$ & 1.64 & $\mathrm{~m}$ \\
$l_{f}$ & 1.04 & $\mathrm{~m}$ \\
$\omega_{r}$ & 47,600 & $\mathrm{~N} / \mathrm{rad}$ \\
$m$ & 1481 & $\mathrm{~kg}$ \\
\hline
\end{tabular}



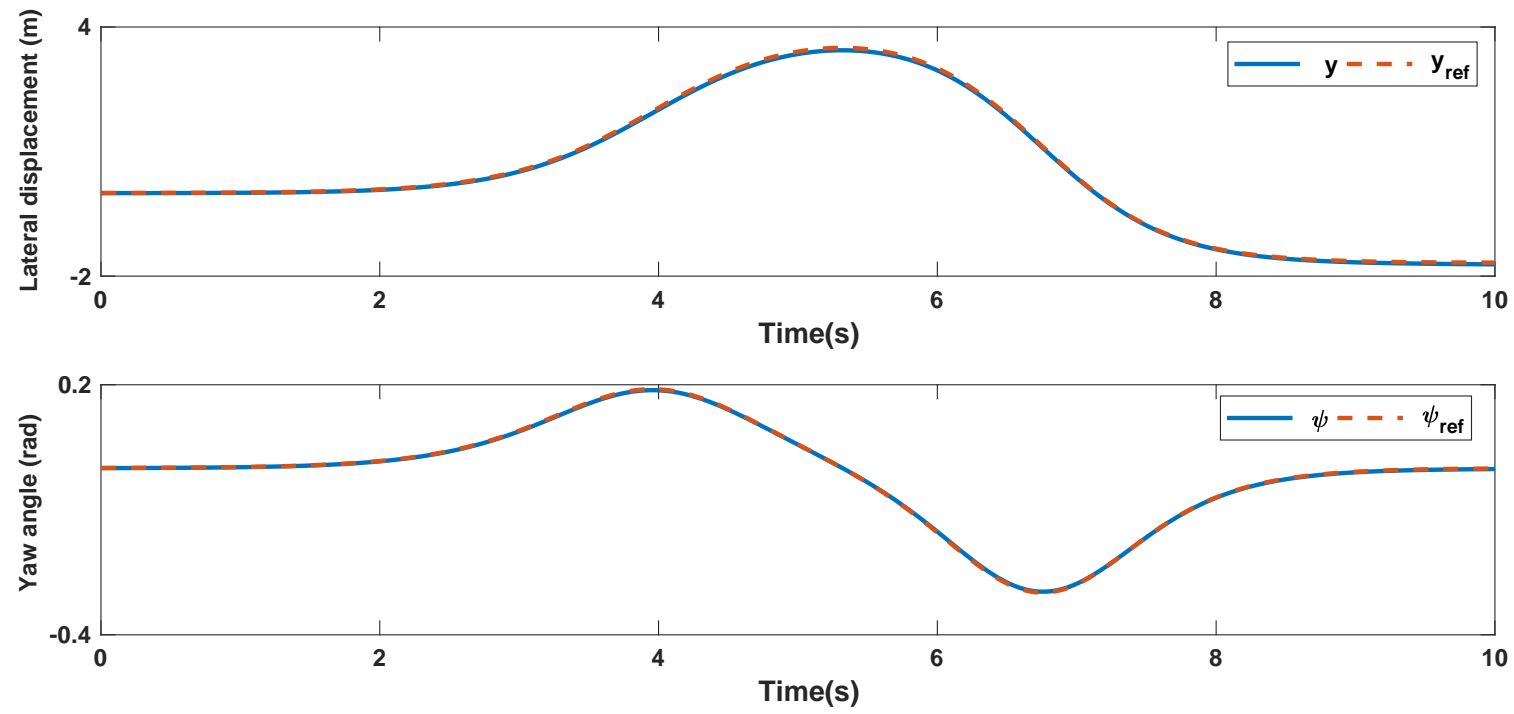

Figure 5. Lateral displacement and Yaw angle.
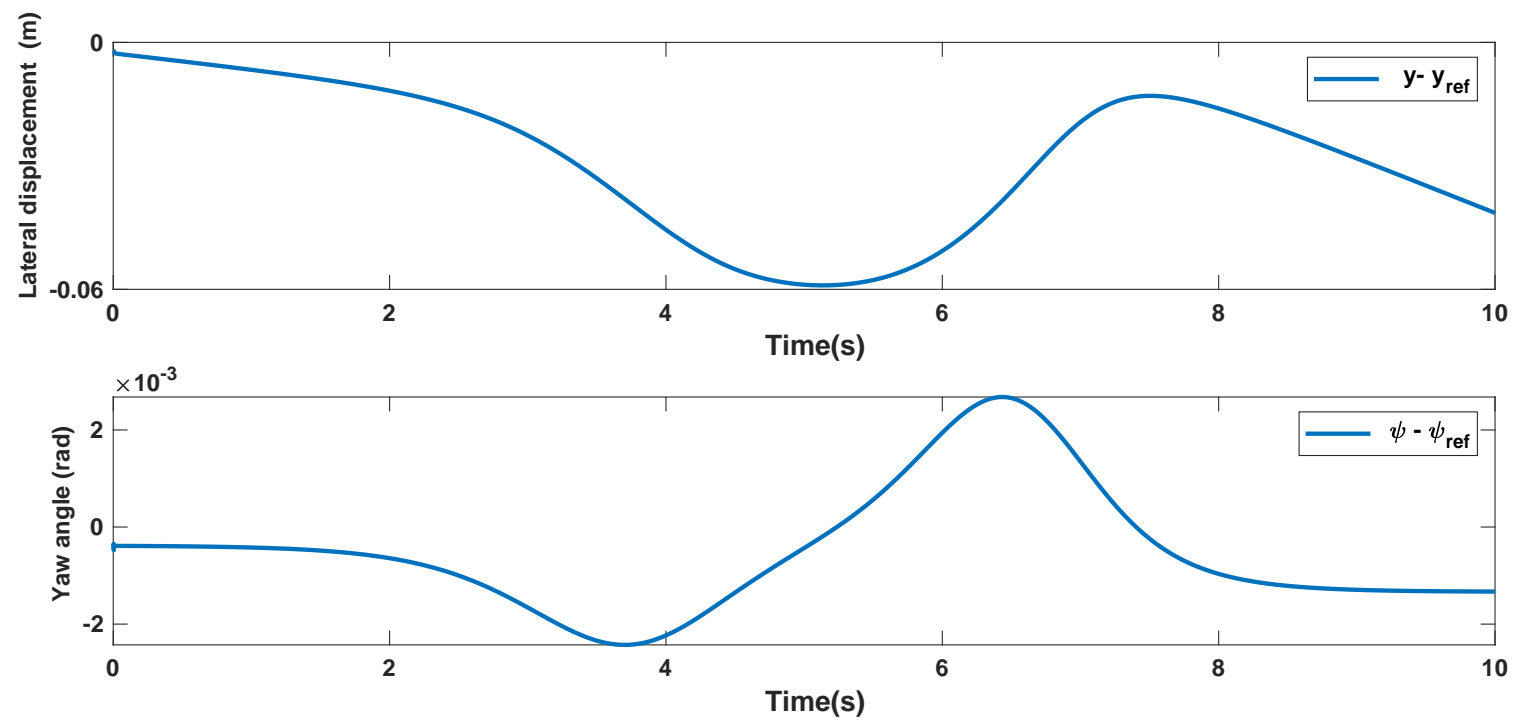

Figure 6. Tracking error.
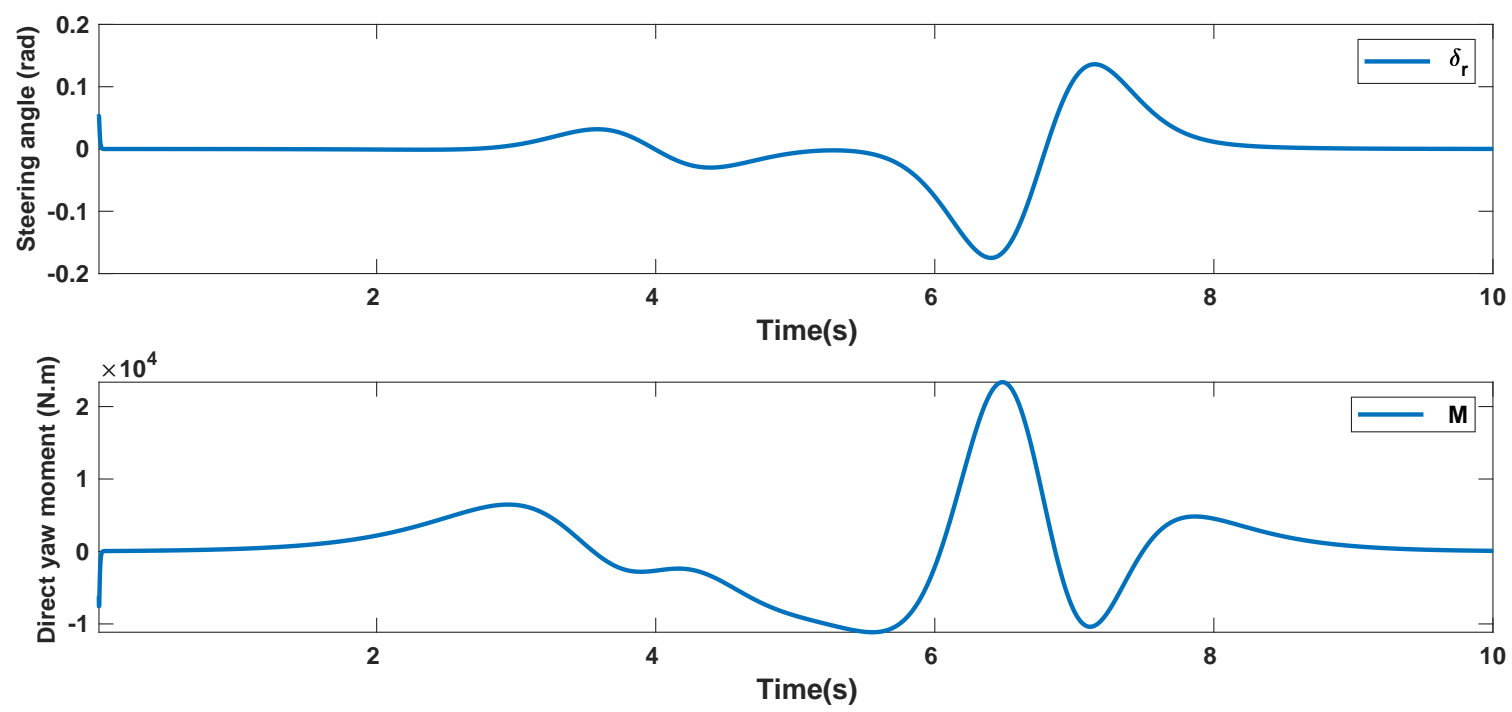

Figure 7. Control signals. 
Table 2. Comparison of RMS and tracking error peak values.

\begin{tabular}{cccccc}
\hline & ADRC [42] & LQT [42] & T2FLC [33] & FLS-MPC [43] & Proposed \\
\hline$y$ & 0.2207 & 1.1049 & 0.0268 & 0.0250 & 0.0241 \\
Maximum of $e_{y}$ & 0.5592 & 1.7757 & 0.0451 & 0.0432 & 0.0317 \\
$\psi$ & 0.0178 & 0.0592 & 0.0014 & 0.0015 & $1.2 \times 10^{-4}$ \\
Maximum of $e_{\psi}$ & 0.0363 & 0.1456 & 0.0017 & 0.0015 & $1.2 \times 10^{-4}$ \\
\hline
\end{tabular}

\subsection{Effect of Longitudinal Velocity}

In this section, the effect of longitudinal velocity is investigated. The longitudinal velocity has a strong effect on the stability and lateral displacement. $\vartheta_{x}$ is changed from $20 \mathrm{~m} / \mathrm{s}$ to $30 \mathrm{~m} / \mathrm{s}$. Similarly, the performance of lane changing maneuver is investigated. The results are depicted in Figures 8 and 9. We see that the maneuver is well completed within the desired time.

A similar comparison is done with other controllers in Table 3. The effect of various velocities on RMS and maximum of errors are described in Table 3. We see that the suggested controller well tackle the effect of changes of longitudinal velocity.

\subsection{Effect of Disturbances}

In this section, the effect of cornering stiffness is investigated. The cornering stiffness for front/rear tires is changed form its nominal value. Based on (4), the uncertainties can also include the other disturbances such as icy or dry road with different adhesion. The various disturbances are taken into account in Table 4, such as random, pulse-shaped and sinusoidal variation of cornering stiffness. The comparison is described in Table 5. We see that the suggested control approach well resist against changes of cornering stiffness.

Table 3. Comparison under different velocities.

\begin{tabular}{|c|c|c|c|}
\hline & $10 \mathrm{~m} / \mathrm{s}$ & $20 \mathrm{~m} / \mathrm{s}$ & $30 \mathrm{~m} / \mathrm{s}$ \\
\hline$y$ & 0.0241 & 0.0338 & 0.0413 \\
\hline Maximum of $e_{y}$ & 0.0317 & 0.0498 & 0.0531 \\
\hline$\psi$ & $1.2 \times 10^{-4}$ & $1.3 \times 10^{-4}$ & $1.3 \times 10^{-4}$ \\
\hline Maximum of $e_{\psi}$ & $1.1 \times 10^{-4}$ & $1.5 \times 10^{-4}$ & $1.7 \times 10^{-4}$ \\
\hline
\end{tabular}

Table 4. Different external disturbances.

case 1 :

case 2 :

case 3 :
Changes of $c_{f} / c_{r}$ as $c_{f}=c_{f}+5000 \sin (2 t)$

Changes of $c_{f} / c_{r}$ as $c_{f}=c_{f}+10,000 \exp (1-2 t)$

A pulse signal at time $2.5 \mathrm{~s}$ with width 0.25

Table 5. Comparison under various disturbances.

\begin{tabular}{ccccc}
\hline & Normal & Case 1 & Case 2 & Case 3 \\
\hline$y$ & 0.0338 & 0.0339 & 0.0341 & 0.0343 \\
Maximum of $e_{y}$ & 0.0317 & 0.0401 & 0.0451 & 0.0527 \\
$\psi$ & $1.30 \times 10^{-4}$ & $1.32 \times 10^{-4}$ & $1.41 \times 10^{-4}$ & $1.47 \times 10^{-4}$ \\
Maximum of $e_{\psi}$ & $1.50 \times 10^{-4}$ & $1.53 \times 10^{-4}$ & $1.61 \times 10^{-4}$ & $1.66 \times 10^{-4}$ \\
\hline
\end{tabular}



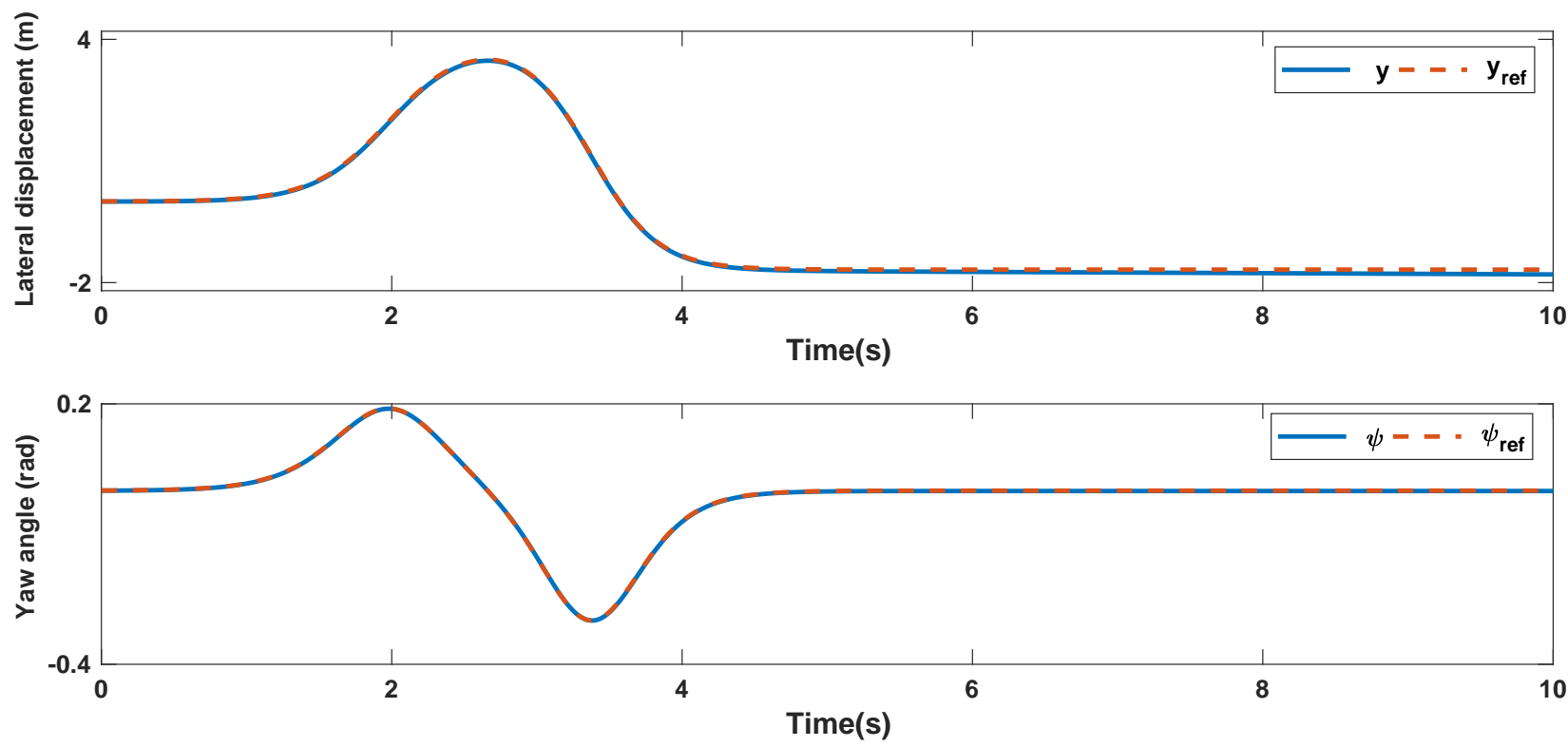

Figure 8. Lateral displacement and Yaw angle, with $\vartheta_{x}=20 \mathrm{~m} / \mathrm{s}$.
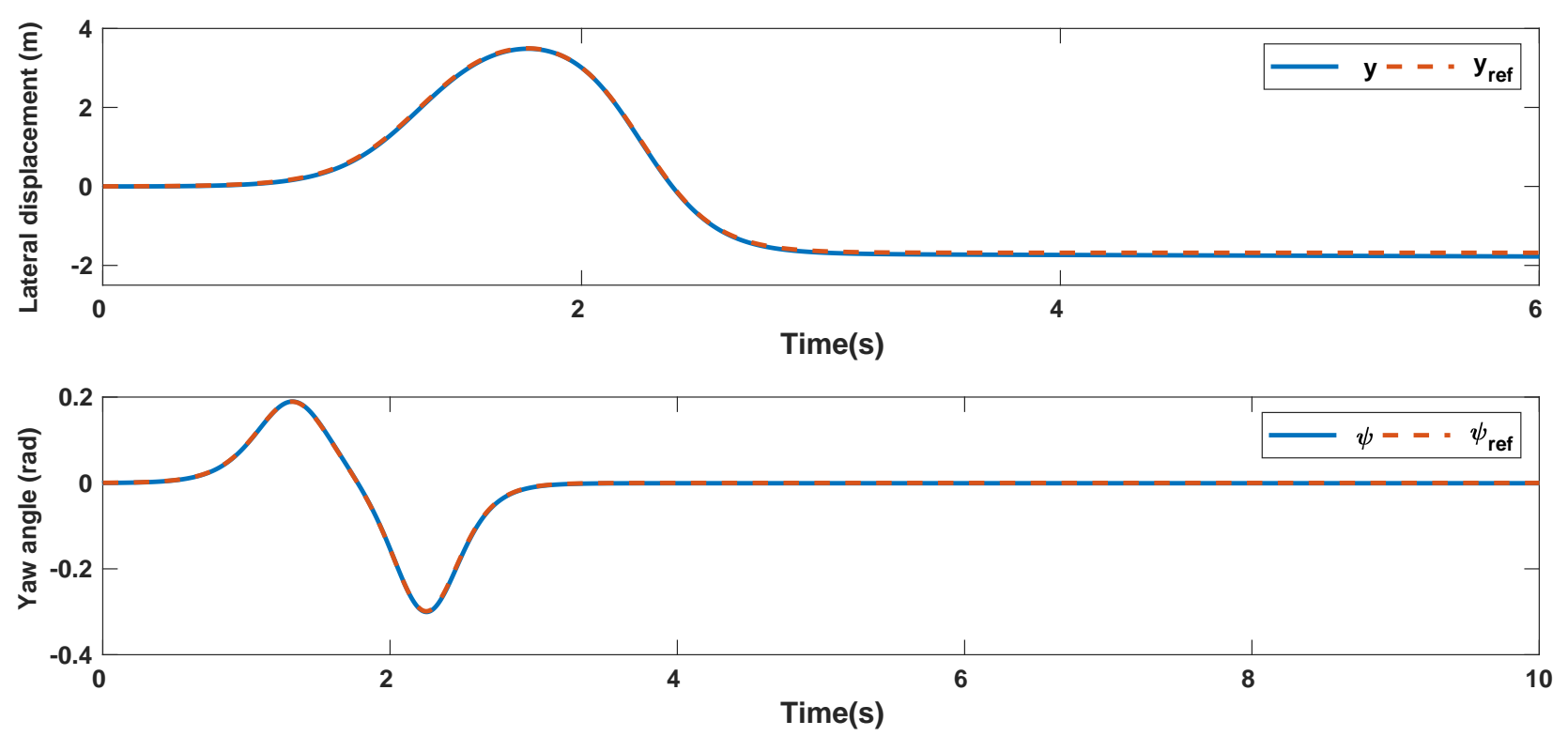

Figure 9. Lateral displacement and Yaw angle, with $\vartheta_{x}=30 \mathrm{~m} / \mathrm{s}$.

\section{Conclusions}

In this study, a new T3FLS-based controller is developed for ARVs. Unlike most studies, the suggested controller does not depend on the mathematical model of ARV, but it is optimized online through the adaptation rules with guaranteed stability. Furthermore, the robustness against perturbations such as variable speed and critical driving maneuvers is ensured by the designed compensators. In various conditions the maneuver capability is examined. For the first examination, the longitudinal velocity is considered to be $10 \mathrm{~m} / \mathrm{s}$ and the performance of changing of heading angle and lateral displacement is investigated. It is shown that the ARV track the desired path with an acceptable lateral displacement. For the second examination, the longitudinal velocity is changed from $20 \mathrm{~m} / \mathrm{s}$ to $30 \mathrm{~m} / \mathrm{s}$. Similarly, the performance of lane changing maneuver is investigated. It is shown that, the suggested controller well resist against changing of longitudinal velocity. For the third scenario, the effect of cornering stiffness is studied. The cornering stiffness for front/rear tires is changed form its nominal value and the better accuracy and robustness of the 
designed controller is demonstrated. In all examinations the accuracy is compared with T2FLC, LQT and ADRC methods and the superiority of designed scheme is shown.

Author Contributions: Conceptualization, A.M., S.M. and W.A.; Data curation, M.-W.T., S.-R.Y., J.T. and M.T.V.; Formal analysis, M.-W.T., S.-R.Y., A.M., J.T., S.M., R.S., W.A., M.T.V. and A.Z.; Funding acquisition, M.-W.T.; Investigation, S.-R.Y., A.M., S.M., W.A. and A.Z.; Methodology, M.-W.T., S.-R.Y., J.T., R.S., W.A. and M.T.V.; Resources, R.S.; Software, and R.S.; Supervision, S.M. and M.T.V.; Validation, A.Z.; Visualization, A.Z.; Writing-original draft, A.M. and J.T. All authors have read and agreed to the published version of the manuscript.

Funding: The research is partially funded by the Ministry of Science and Higher Education of the Russian Federation as part of World-class Research Center program: Advanced Digital Technologies (contract No. 075-15-2020-903 dated 16 November 2020).

Institutional Review Board Statement: Not applicable.

Informed Consent Statement: Not applicable.

Data Availability Statement: The study do not report any data.

Acknowledgments: The authors would like to express their sincere thanks to Sakthivel Rathinasamy for his help in improving this paper.

Conflicts of Interest: The authors declare no conflicts of interest.

\section{References}

1. Jin, X.; Yang, J.; Li, Y.; Zhu, B.; Wang, J.; Yin, G. Online estimation of inertial parameter for lightweight electric vehicle using dual unscented Kalman filter approach. IET Intell. Transp. Syst. 2020, 14, 412-422. [CrossRef]

2. Tian, M.W.; Talebizadehsardari, P. Energy cost and efficiency analysis of building resilience against power outage by shared parking station for electric vehicles and demand response program. Energy 2021, 215, 119058. [CrossRef]

3. Jin, X.; Wang, J.; Sun, S.; Li, S.; Yang, J.; Yan, Z. Design of constrained robust controller for active suspension of in-wheel-drive electric vehicles. Mathematics 2021, 9, 249. [CrossRef]

4. Chen, C.H.; Jeng, S.Y.; Lin, C.J. Mobile robot wall-following control using fuzzy logic controller with improved differential search and reinforcement learning. Mathematics 2020, 8, 1254. [CrossRef]

5. Chen, C.H.; Jeng, S.Y.; Lin, C.J. Using an Adaptive Fuzzy Neural Network Based on a Multi-Strategy-Based Artificial Bee Colony for Mobile Robot Control. Mathematics 2020, 8, 1223. [CrossRef]

6. Nakata, R.; Tanemura, M.; Chida, Y.; Mitsuhashi, T. Undershoot response analysis of circular path-following control of an autonomous vehicle. Mech. Eng. J. 2021, 8, 21-00002. [CrossRef]

7. Liang, X.; Qu, X.; Hou, Y.; Li, Y.; Zhang, R. Finite-time unknown observer based coordinated path-following control of unmanned underwater vehicles. J. Frankl. Inst. 2021, 358, 2703-2721. [CrossRef]

8. Huang, C.; Zhang, X.; Zhang, G. Decentralized event-triggered cooperative path-following control for multiple autonomous surface vessels under actuator failures. Appl. Ocean. Res. 2021, 113, 102751. [CrossRef]

9. Qu, X.; Liang, X.; Hou, Y.; Li, Y.; Zhang, R. Path-following control of unmanned surface vehicles with unknown dynamics and unmeasured velocities. J. Mar. Sci. Technol. 2021, 26, 395-407. [CrossRef]

10. Wang, S.; Levin, M.W.; Caverly, R.J. Optimal parking management of connected autonomous vehicles: A control-theoretic approach. Transp. Res. Part C Emerg. Technol. 2021, 124, 102924. [CrossRef]

11. Yoon, Y.; Kim, C.; Lee, J.; Yi, K. Interaction-Aware Probabilistic Trajectory Prediction of Cut-In Vehicles Using Gaussian Process for Proactive Control of Autonomous Vehicles. IEEE Access 2021, 9, 63440-63455. [CrossRef]

12. Huang, J.; Wang, W.; Su, X. Adaptive iterative learning control of multiple autonomous vehicles with a time-varying reference under actuator faults. IEEE Trans. Neural Netw. Learn. Syst. 2021,1-14.

13. Xiao, W.; Cassandras, C.G.; Belta, C.A. Bridging the gap between optimal trajectory planning and safety-critical control with applications to autonomous vehicles. Automatica 2021, 129, 109592. [CrossRef]

14. Zhao, B.; Zhang, X.; Liang, C. A novel path-following control algorithm for surface vessels based on global course constraint and nonlinear feedback technology. Appl. Ocean. Res. 2021, 111, 102635. [CrossRef]

15. Cao, X.; Tian, Y.; Ji, X.; Qiu, B. Fault-Tolerant Controller Design for Path Following of the Autonomous Vehicle under the Faults in Braking Actuators. IEEE Trans. Transp. Electrif. 2021, 7, 2530-2540. [CrossRef]

16. Sanchez, I.; D’Jorge, A.; Raffo, G.V.; González, A.H.; Ferramosca, A. Nonlinear Model Predictive Path Following Controller with Obstacle Avoidance. J. Intell. Robot. Syst. 2021, 102, 1-18. [CrossRef]

17. Holovatenko, I.; Pysarenko, A. Energy-Efficient Path-Following Control System of Automated Guided Vehicles. J. Control Autom. Electr. Syst. 2021, 32, 390-403. [CrossRef]

18. Xie, J.; Xu, X.; Wang, F.; Tang, Z.; Chen, L. Coordinated control based path following of distributed drive autonomous electric vehicles with yaw-moment control. Control Eng. Pract. 2021, 106, 104659. [CrossRef] 
19. Yañez-Badillo, H.; Tapia-Olvera, R.; Beltran-Carbajal, F. Adaptive Neural Motion Control of a Quadrotor UAV. Vehicles 2020, 2, 468-490. [CrossRef]

20. Yañez-Badillo, H.; Beltran-Carbajal, F.; Tapia-Olvera, R.; Favela-Contreras, A.; Sotelo, C.; Sotelo, D. Adaptive Robust Motion Control of Quadrotor Systems Using Artificial Neural Networks and Particle Swarm Optimization. Mathematics $2021,9,2367$. [CrossRef]

21. Napole, C.; Barambones, O.; Calvo, I.; Derbeli, M.; Silaa, M.Y.; Velasco, J. Advances in Tracking Control for Piezoelectric Actuators Using Fuzzy Logic and Hammerstein-Wiener Compensation. Mathematics 2020, 8, 2071. [CrossRef]

22. Tian, M.; Yan, S.; Tian, X. Discrete approximate iterative method for fuzzy investment portfolio based on transaction cost threshold constraint. Open Phys. 2019, 17, 41-47. [CrossRef]

23. Derbeli, M.; Napole, C.; Barambones, O. Machine Learning Approach for Modeling and Control of a Commercial Heliocentris FC50 PEM Fuel Cell System. Mathematics 2021, 9, 2068. [CrossRef]

24. Napole, C.; Derbeli, M.; Barambones, O. Fuzzy Logic Approach for Maximum Power Point Tracking Implemented in a Real Time Photovoltaic System. Appl. Sci. 2021, 11, 5927. [CrossRef]

25. Tork, N.; Amirkhani, A.; Shokouhi, S.B. An adaptive modified neural lateral-longitudinal control system for path following of autonomous vehicles. Eng. Sci. Technol. Int. J. 2021, 24, 126-137.

26. Wang, M.; Chen, J.; Deng, Z.; Xiang, Y. Velocity Planning Method Base on Fuzzy Neural Network for Autonomous Vehicle. IEEE Access 2021, 9, 19111-19126. [CrossRef]

27. Yang, Z.; Huang, J.; Yang, D.; Zhong, Z. Design and Optimization of Robust Path Tracking Control for Autonomous Vehicles with Fuzzy Uncertainty. IEEE Trans. Fuzzy Syst. 2021, 1. [CrossRef]

28. Li, M.; Long, Y.; Li, T.; Bai, W. Observer-Based Adaptive Fuzzy Event-Triggered Path Following Control of Marine Surface Vessel. Int. J. Fuzzy Syst. 2021, 23, 2021-2036. [CrossRef]

29. Lin, F.; Wang, S.; Zhao, Y.; Cai, Y. Research on autonomous vehicle path tracking control considering roll stability. Proc. Inst. Mech. Eng. Part D J. Automob. Eng. 2021, 235, 199-210. [CrossRef]

30. Wu, Q.; Cheng, S.; Li, L.; Yang, F.; Meng, L.J.; Fan, Z.X.; Liang, H.W. A fuzzy-inference-based reinforcement learning method of overtaking decision making for automated vehicles. Proc. Inst. Mech. Eng. Part D J. Automob. Eng. 2021, 09544070211018099. [CrossRef]

31. Wei, L.; Wang, X.; Li, L.; Fan, Z.; Dou, R.; Lin, J. TS fuzzy model predictive control for vehicle yaw stability in nonlinear region. IEEE Trans. Veh. Technol. 2021, 70, 7536-7546. [CrossRef]

32. Xu, X.; Su, P.; Wang, F.; Chen, L.; Xie, J.; Atindana, V.A. Coordinated control of dual-motor using the interval type-2 fuzzy logic in autonomous steering system of AGV. Int. J. Fuzzy Syst. 2021, 23, 1070-1086. [CrossRef]

33. Mohammadzadeh, A.; Taghavifar, H. A robust fuzzy control approach for path-following control of autonomous vehicles. Soft Comput. 2020, 24, 3223-3235. [CrossRef]

34. Yao, L.; Pitla, S.K.; Zhao, C.; Liew, C.; Hu, D.; Yang, Z. An Improved Fuzzy Logic Control Method for Path Tracking of an Autonomous Vehicle. Trans. ASABE 2020, 63, 1895-1904. [CrossRef]

35. Nabipour, N.; Qasem, S.N.; Jermsittiparsert, K. Type-3 fuzzy voltage management in PV/hydrogen fuel cell/battery hybrid systems. Int. J. Hydrogen Energy 2020, 45, 32478-32492. [CrossRef]

36. Liu, Z.; Mohammadzadeh, A.; Turabieh, H.; Mafarja, M.; Band, S.S.; Mosavi, A. A New Online Learned Interval Type-3 Fuzzy Control System for Solar Energy Management Systems. IEEE Access 2021, 9, 10498-10508. [CrossRef]

37. Ma, C.; Mohammadzadeh, A.; Turabieh, H.; Mafarja, M.; Band, S.S.; Mosavi, A. Optimal Type-3 Fuzzy System for Solving Singular Multi-Pantograph Equations. IEEE Access 2020, 8, 225692-225702. [CrossRef]

38. Vafaie, R.H.; Mohammadzadeh, A.; Piran, M. A new type-3 fuzzy predictive controller for MEMS gyroscopes. Nonlinear Dyn. 2021, 106, 381-403. [CrossRef]

39. Cao, Y.; Raise, A.; Mohammadzadeh, A.; Rathinasamy, S.; Band, S.S.; Mosavi, A. Deep learned recurrent type-3 fuzzy system: Application for renewable energy modeling/prediction. Energy Rep. 2021, in press. [CrossRef]

40. Mohammadzadeh, A.; Sabzalian, M.H.; Zhang, W. An interval type-3 fuzzy system and a new online fractional-order learning algorithm: Theory and practice. IEEE Trans. Fuzzy Syst. 2019, 28, 1940-1950. [CrossRef]

41. Falcone, P.; Borrelli, F.; Asgari, J.; Tseng, H.E.; Hrovat, D. Predictive active steering control for autonomous vehicle systems. IEEE Trans. Control Syst. Technol. 2007, 15, 566-580. [CrossRef]

42. Xia, Y.; Pu, F.; Li, S.; Gao, Y. Lateral path tracking control of autonomous land vehicle based on ADRC and differential flatness. IEEE Trans. Ind. Electron. 2016, 63, 3091-3099. [CrossRef]

43. Hwang, C.-L.; Yang, C.-C.; Hung, J.Y. Path Tracking of an Autonomous Ground Vehicle with Different Payloads by Hierarchical Improved Fuzzy Dynamic Sliding-Mode Control. IEEE Trans. Fuzzy Syst. 2018, 26, 899-914. [CrossRef] 